\title{
АРГУМЕНТ ПОСЛЕДСТВИЙ КАК МОДИФИКАЦИЯ ЛЕНИВОГО СОФИЗМА
}

\section{THE CONSEQUENCE ARGUMENT AS A MODIFICATION OF THE LAZY REASON}

A. Yurasov

Summary: The consequence argument in defense of incompatibilism has a sophistic nature, namely, it is a modification of the so-called lazy reason. The connection of the consequence argument with lazy reason is mediated by the rule $\beta$. Even if the relation "up to us" is understood as not determined unambiguously by external causes, the consequences of phenomena that are not up to us may be up to us. The fact that the consequence argument is considered in analytical philosophy as the main incompatibilist argument indicates a crisis in modern studies of free will.

Keywords: consequence argument, free will, incompatibilism, lazy reason, determinism, rule $\beta$.

\section{Введение}

И нкомпатибилизм - теоретическая позиция, в соответствии с которой свобода воли несовместима с детерминизмом (как правило, подразумевается каузальный детерминизм). Самым известным инкомпатибилистским аргументом является аргумент последствий, сформулированный Карлом Жине [6, с. 87-104], Дэвидом Виггинсом [10, с. 31-62] и Питером ван Инвагеном [9, с. 185-199; 8]. «Версия ван Инвагена получила наибольшее распространение. Обычно, говоря об аргументе последствий, имеют в виду именно ее. В общем виде аргумент звучит следующим образом: “Если детерминизм истинен, то наши действия являются последствиями законов природы и событий в отдаленном прошлом. Однако от нас не зависит то, что происходило до нашего рождения, и также не зависит, каковы законы природы. Следовательно, их последствия (включая наши действия в настоящем) от нас не зависят"» [3, с. 88]. Данный аргумент представляет собой видоизменение древнего софизма, известного под названием ленивый софизм.

\section{Ложность аргумента}

Так называемое правило $\beta$, которое берется в качестве одной из посылок аргумента, заключается в том, что от нас не зависят последствия того, что от нас не зависит. Ложность этого правила очевидна, ибо если некоторый не зависящий от нас фактор обусловливает нашу волю, то результат наших действий зависит как от этого фактора, так и от нас. Если же под словами «зависеть от»

\author{
Юрасов Андрей Александрович \\ К.ф.н., президент, Благотворительный фонд \\ «Чувство дома» \\ yurasov@chuvstvo-doma.ru
}

Аннотация: Аргумент последствий в защиту инкомпатибилизма имеет софистическую природу, а именно представляет собой модификацию так называемого ленивого софизма. Связь аргумента последствий с ленивым софизмом опосредована правилом . Даже если отношение «зависит от нас» понимается как не определенное однозначно внешними причинами, последствия не зависящих от нас феноменов могут зависеть от нас. Тот факт, что аргумент последствий рассматривается в аналитической философии в качестве главного инкомпатибилисткого аргумента, свидетельствует 0 кризисе в современных исследованиях свободы воли.

Ключевые слова: аргумент последствий, свобода воли, инкомпатибилизм, ленивый софизм, детерминизм, правило $\beta$.

подразумевается отношение, которое однозначно не обусловлено никакими предшествующими событиями и законами природы, то такое отношение несовместимо с каузальным детерминизмом просто потому, что он предполагает, что некоторые события (в том числе внешние по отношению к нам, нашим ценностям) в совокупности с законами природы однозначно определяют нашу волю. В.В.Васильев справедливо говорит об аргументе последствий, что он «вызвал много дискуссий, но едва ли заслуженно» [1, с. 28], а также, что «если наши действия причиняются нашими желаниями, то они зависят от этих желаний и от нас, их субъектов. И зависимость этих действий от желаний не противоречит тому, что сами желания детерминируются отдаленными причинами. Из того, что причина события имеет причину, не следует, что она из-за этого перестает быть причиной. Подобные утверждения кажутся просто нелепостью. Единственная трактовка аргумента ван Инвагена, при которой он не выглядит абсурдным, состоит в том, что при детерминизме события предопределены и в этом смысле не зависят от нас. Но это вытекает из дефиниции детерминизма, так что тут не нужно дополнительной аргументации» [1, с. 30].

Стоит отметить, что если понимать термин «последствие» в обычном смысле, то аргумент последствий не работает даже в том случае, если под отношением «зависит от» подразумевается ничем однозначно не обусловленная зависимость. Если некоторое событие $X$, не зависящее от меня, вызывает событие $Y$ лишь при условии, что я этому не помешаю, то $\mathrm{Y}$ является последствием $\mathrm{X}$, 
когда я этому не мешаю. Даже если мое вмешательство или невмешательство не детерминировано ничем внешним и зависит только от меня, Y будет последствием $\mathrm{X}$, если я этому не помешаю. Поэтому последствие события, которое от меня не зависит, может от меня зависеть. Конечно, можно сказать, что $Y$ является последствием того, что имеют место и событие $X$, и мое решение о невмешательстве. Однако это не противоречит тому, что $\mathrm{Y}$ является и последствием одного только $\mathrm{X}$ : можно обратить внимание на то, что если событие $X$ сочетается $c$ моим решением о невмешательстве, то это не является достаточным условием возникновения Ү, как не является таковым и событие $X$, рассмотренное само по себе.

Чтобы это рассуждение легче воспринималось, проиллюстрируем его примером. Я наблюдаю медленное движение желтого шара по направлению к красному. У меня есть возможность, протянув руку, зафиксировать положение красного шара так, что, когда произойдет его столкновение с желтым, красный шар не сдвинется с места. Но если я этого не сделаю, то красный шар будет сбит желтым. Предположим также, что у меня нет возможности повлиять на движение желтого шара вплоть до момента его столкновения с красным и что я принимаю решение не фиксировать положение красного шара. Тогда движение желтого шара (событие X) не зависит от меня, но его последствие - изменение положения красного шара (событие Y) - от меня зависит, ведь у меня была возможность предотвратить это событие. Причем это верно, даже если мое решение не прикасаться к красному шару однозначно не определено внешними факторами. Странно было бы не называть в рассматриваемой ситуации изменение положения красного шара последствием движения желтого шара. Но изменение положения красного шара очевидно зависит от моего решения, несмотря на то, что движение желтого шара от меня не зависит.

\section{Бесполезная формализашия}

Аргументу последствий посвящена большая литература, и прежде всего в связи с тем, что ван Инваген представил его в формализованном виде (в различных версиях, из которых наиболее известна та, в которой применяется модальная логика). Сходную структуру имеет и прямой аргумент, также сформулированный ван Инвагеном и направленный на обоснование инкомпатибилизма напрямую, без апеллирования к невозможности поступать иначе. Эти формализмы не только бесполезны, но и способствуют путанице. Имеет смысл продемонстрировать это на примере ошибки, допущенной ван Инвагеном именно в связи с формализацией доказательства. Версия аргумента последствий, названная основным аргументом, изложена применительно к случаю некоего судьи J, который на судебном разбирательстве в момент Т имел возможность поднять руку, проголосовав тем самым за помилование преступника, однако этого не сделал, и тот был казнен. Обозначения: $\mathrm{T}_{0}$ - момент времени до рождения J, $\mathrm{P}_{0}$ - пропозиция, выражающая состояние мира в момент $\mathrm{T}_{0^{\prime}} \mathrm{P}$ - пропозиция, выражающая состояние мира в момент T, L - конъюнкция всех законов физики. Основной аргумент:

«(1) Если детерминизм истинен, то из конъюнкции L и $\mathrm{P}_{0}$ следует $\mathrm{P}$.

(2) Если бы J поднял руку в момент Т, то P была бы ложной.

(3) Если (2) верно, то если J мог поднять руку в T, то J мог бы сделать Р ложной.

(4) Если J мог сделать Р ложной и если из конъюнкции $\mathrm{L}$ и $\mathrm{P}_{0}$ следует $\mathrm{P}$, то J мог сделать конъюнкцию $\mathrm{L}$ и $\mathrm{P}_{0}$ ложной.

(5) Если J мог сделать конъюнкцию $L$ и $\mathrm{P}_{0}$ ложной, то J мог сделать L ложной.

(6) J не мог сделать L ложной.

(7) Если детерминизм истинен, то J не мог поднять руку в Т» $[9$, с. 191].

Ван Инваген утверждает, что этот аргумент верен и в случае условного анализа («мог сделать» означает «сделал бы, если бы выбрал так сделать»), и поясняет это на примере шестого пункта: если J выбрал бы сделать L ложной, то не смог бы этого сделать (ведь не во власти человека изменять законы природы). Ошибка ван Инвагена состоит, во-первых, в том, что он рассматривает действие «сделать $L$ ложной» как объект выбора, вовторых, в игнорировании того, что если бы J захотел поднять руку, то конъюнкция $L$ и $P_{0}$ была бы ложной. Таким образом, очевидные вещи стали трудными для понимания в результате ненужной формализации, что привело к софистическим выводам.

Ложность аргумента последствий легко устанавливается без обращения к его формализованным версиям. Но если ошибочна сама идея аргумента, то никакая ее формализация не в состоянии этого исправить. Напротив, она лишь неоправданно усложняет аргумент и создает видимость научного подхода.

\section{ヘенивый софизм}

Аргумент последствий представляет собой модификацию ленивого софизма: «...если будущее необходимо, то произойдет то, что должно произойти, как бы я ни поступал» [2, с. 54]. Родственность аргумента последствий ленивому софизму почему-то упускают из виду, несмотря на обилие литературы, посвященной этому аргументу. В его основе лежит правило $\beta$, которое заключается в том, что от нас не зависят последствия того, что от нас не зависит. Разумеется, это правило полностью обесценивает аргумент.

Ленивый софизм был опровергнут еще в древности. 
«Это умозаключение опровергается Хрисиппом. Среди [высказываний о] событиях, говорит он, одни простые [необусловленные] (simplicia), а другие - связанные [обусловленные]. Простое событие [выражено в высказывании] "Сократ умрет в такой-то день": день смерти для него определен и не зависит от того, сделает он что-нибудь или не сделает. А вот если предсказано: "У Лая родится Эдип", то тут уже нельзя добавить: “Сойдется ли Лай с женой или не сойдется", - ведь это события связанные и потому соопределенные. А называет он их так, поскольку было назначено, что Лай должен был сойтись с женой и от этого предстояло родиться Эдипу» [4, с. 157, 158].

Если законы природы и факты определенного момента в далеком прошлом однозначно определяют все последующие события, то это не означает, что мои действия от меня не зависят. Напротив, мои решения влияют на мои действия.

\section{Зак^ючение}

В Стэнфордской философской энциклопедии указано, что аргумент последствий наряду с идеями Питера Стросона и Гарри Франкфурта радикально изменил дискуссию о свободе воли [5]. Настораживает тот факт, что в современных дискуссиях по этой великой проблеме одной из ключевых тем является аргумент, софистическая природа которого очевидна. Представление о том, что он является весомым, широко распространено: «С момента своего первого появления аргумент последствий был единственным наиболее влиятельным соображением в пользу тезиса о том, что свобода воли, понимаемая в терминах свободы выбора, несовместима с детерминизмом» [7, с. 72]. Это не может не свидетельствовать о кризисе современной философии свободной воли.

\section{ЛИТЕРАТУРА}

1. Васильев В.В. В защиту классического компатибилизма. Эссе о свободе воли. М.: ЛЕНАНД, 2017. — 200 c.

2. Лейбниц Г.В. Опыты теодицеи о благости Божией, свободе человека и начале зла // Лейбниц Г. В. Сочинения в четырех томах. Т. 4. М.: Мысль, 1989. — 554 с.

3. Мишура А.С. Аргумент последствий в дискуссии о свободе воли. // Мысль. Выпуск 21 (2016). - 186 с.

4. Цицерон М.Т. 0 судьбе // Фрагменты ранних стоиков. Т. 2, ч. 2. М.: Греко-латинский кабинет Ю.А. Шичалина, 2002. - 272 с.

5. Compatibilism // The Stanford Encyclopedia of Philosophy. URL: https://plato.stanford.edu/entries/compatibilism/ (дата обращения: 28.07.2021).

6. Ginet C. Might We Have No Choice? // Freedom and Determinism. New York: Random House, 1966. — 207 p.

7. McKenna M., Pereboom D. Free Will. A Contemporary Introduction. New York and London: Routledge, 2016. — 329 p.

8. Van Inwagen P. An Essay on Free Will. Oxford: Clarendon Press, 1983. - $248 \mathrm{p}$.

9. Van Inwagen P. The Incompatibility of Free Will and Determinism // Philosophical Studies. Vol. 27, №3, Mar. 1975. — 220 p.

10. Wiggins D. Towards a Reasonable Libertarianism // Essays on Freedom of Action. London and Boston: Routledge and Kegan Paul, 1973. — 215 p. 